\title{
2 Research Square \\ Predicting Amyloid Pathology in Mild Cognitive Impairment Using Radiomics Analysis of Magnetic Resonance Imaging
}

\section{Yae Won Park}

Yonsei University College of Medicine

\section{Dongmin Choi}

Yonsei University

Mina Park ( $\nabla$ to.minapark@yuhs.ac)

Gangnam Severance Hospital https://orcid.org/0000-0002-2005-7560

\section{Sung Jun Ahn}

Yonsei University College of Medicine

\section{Sung Soo ahn}

Yonsei University College of Medicine

\section{Sang Hyun Suh}

Yonsei University College of Medicine

\section{Seung-Koo Lee}

Yonsei University College of Medicine

\section{Research}

Keywords: amyloid, artificial intelligence, machine learning, mild cognitive impairment, radiomics

Posted Date: March 10th, 2020

DOI: https://doi.org/10.21203/rs.3.rs-16481/v1

License: (9) (i) This work is licensed under a Creative Commons Attribution 4.0 International License.

Read Full License

Version of Record: A version of this preprint was published at Journal of Alzheimer's Disease on January 19th, 2021. See the published version at https://doi.org/10.3233/JAD-200734. 


\section{Abstract}

Background: Noninvasive identification of amyloid $\beta(A \beta)$ is important in mild cognitive impairment (MCl) patients for better clinical management. This study aimed to evaluate whether radiomics features in the hippocampus in $\mathrm{MCl}$ improve the prediction of cerebrospinal fluid (CSF) A 42 status when integrated with clinical and genetic profiles.

Methods: A total of $407 \mathrm{MCl}$ subjects from the Alzheimer's Disease Neuroimaging Initiative were allocated to the training $(n=324)$ and test $(n=83)$ sets. Radiomics features $(n=214)$ from the bilateral hippocampi were extracted from T1-weighted images of magnetic resonance imaging (MRI). A previously defined cutoff ( $<192 \mathrm{pg} / \mathrm{mL}$ ) was applied for CSF A 42 status. After feature selection, random forest with subsampling methods were trained to predict the CSF A 42 with three models: 1) a radiomics model; 2) a clinical model based on clinical and genetic profiles including demographics, APOE $\varepsilon 4$ genotype, and neuropsychological tests; and 3) a combined model based on radiomics and clinical profiles. The prediction performance of the classifier was validated in the test set using the area under the receiver operating characteristic curve (AUC).

Results: The radiomics model identified 33 radiomics features to predict CSF A 42 , which showed an AUC of 0.674 in the best performing radiomics model in the test set. The clinical model identified 6 clinical features to predict CSF A 42 , which showed an AUC of 0.758 in the best performing clinical model in the test set. The combined model based on radiomics and clinical profiles identified a total of 37 features (32 from radiomics and 5 from clinical features), showing an AUC of 0.823 in the best performing combined model test set, which showed the highest performance among the three models.

Conclusions: Radiomics model from MRI can help predict CSF A 42 status in $\mathrm{MCl}$ patients and potentially triage the patients for the invasive and costly $A \beta$ test.

\section{Background}

Mild cognitive impairment $(\mathrm{MCl})$ is often considered a prodromal stage of Alzheimer's disease (AD), but patients with $\mathrm{MCl}$ are heterogeneous with different rates of progression toward AD. (1) The identification of $\mathrm{MCl}$ patients at risk for dementia due to $A D$ is of utmost importance for prediction of disease prognosis as well as for potential preventative and therapeutic treatments. (2) Therefore, biomarkerbased detection of the initial amyloid $\beta(A \beta)$ pathology is important for better clinical management of $\mathrm{MCl}$, potentially providing the opportunity to start disease-modifying therapies before the progression of AD.

$A \beta$ pathology can be assessed by measurement of $A \beta$ concentration in the cerebrospinal fluid (CSF) or via molecular imaging techniques such as positron emission tomography (PET) scans using a specific radioligand for $A \beta$. (3) However, obtaining CSF is by lumbar puncture is invasive, and PET scans are costly, invasive due to radiation exposure, and are not always available. (4) Therefore, finding non- 
invasive predictive biomarkers for $A \beta$ status could reduce the number of invasive examinations and financial burden.

Structural neuroimaging using magnetic resonance imaging (MRI) has been shown to be useful in characterizing dementia and cognitive decline due to AD pathology. $(5,6)$ Structural changes in ADvulnerable structures such as the entorhinal cortex, hippocampus, and temporal lobe have been reported to be diagnostic indicators of cognitive impairment and even used for the prediction of amyloid pathology. (6) Compared with CSF study and PET scan, MRI has the advantages of being non-invasive and its expenditure is usually reimbursed in most countries. Therefore, if MRI can predict the A $\beta$ pathology, it can have potential advantages over CSF study or PET scans.

Radiomics is an emerging field that extracts automated quantifications of enormous radiologic phenotype using data characterization algorithms. (7) Because radiomics models use high-throughput imaging features, they likely discover hidden information that is inaccessible with single-parameter approaches. To the best of our knowledge, there has been no previous radiomics study to predict the amyloid pathology in the $\mathrm{MCl}$ population. We hypothesized that radiomics features of brain MRI along with machine learning technique and in addition to clinical and genetic profiles can aid in predicting the CSF $A \beta_{42}$ status in $\mathrm{MCl}$ patients.

\section{Methods}

\section{Patient population}

A total of 494 patients diagnosed with $\mathrm{MCl}$ who were enrolled in the Alzheimer's Disease Neuroimaging Initiative-GO (ADNI-GO) and ADNI2 database (adni.loni.usc.edu) were included in this study. The eligible patients were those who completed baseline visit and underwent MRI. Of these, we excluded those who had 1) missing demographics or neuropsychological (NP) test data $(n=68), 2)$ error in hippocampus masks or severe artifacts on MRI $(n=18)$, and 3) error in radiomics processing $(n=1)$. Finally, 407 patients were enrolled in this study. The enrolled patients were semi-randomly allocated to the training (n $=324)$ and test $(n=83)$ sets, with stratification for CSF A $\beta$ status (Fig. 1).

Apolipoprotein E (APOE) gene polymorphism was assessed and patients were divided into $\varepsilon 4$ carriers $(\varepsilon 4 / \varepsilon 4$ or $\varepsilon 3 / \varepsilon 4)$ and non-carriers according to the presence of the APOE $\varepsilon 4$ allele. NP test results including the Mini-Mental Statue Examination (MMSE), the 11-item Alzheimer's Disease Assessment Scale cognitive subscale (ADAS-cog), and Logical Memory I (LM I) immediate recall and Logical Memory II (LM II) delayed recall from MCl patients were obtained. (8-10) The total numbers of story units recalled in LM I were labeled as the LM I total score. The total number of story units recalled in LM II were labeled as the LM II total score. The total number of cues in LM II were labeled as the LM II cue score. CSF A $\beta_{42}$ was measured for all patients with available CSF samples using the ADNI Biomarker Core at the University of Pennsylvania School of Medicine. (11) CSF $A \beta_{42}$ was dichotomized to $A \beta$ - or $A \beta+$ groups using a previously defined CSF concentration threshold (CSF A $\beta_{42}<192 \mathrm{pg} / \mathrm{mL}$ ). (12) 


\section{MRI acquisition}

MRIs were acquired using a 3-Tesla system as per standardized protocols compatible with the ADNI. (13) T1-weighted images were acquired using an axial three-dimensional spoiled gradient echo sequence. Axial T2 fluid-attenuated inversion recovery images were acquired.

\section{Image postprocessing and radiomics feature extraction}

Automated mask extraction of right and left hippocampus was performed using volBrain (https://volbrain.upv.es/), $(14,15)$ which is a robust automatic pipeline for brain segmentation with high accuracy. (16) After denoising with an adaptive nonlocal mean filter, images were affine-registered in the Montreal Neurological Institute space using Advanced Normalization Tools software, (17) corrected for image inhomogeneities using N4, and, finally, intensity normalized. (18) Then, the hippocampus was segmented based on a multi-atlas framework combining nonlinear registration and patch-based label fusion. (19) Two experienced neuroradiologists (Y.W.P. and M.P, with 8 years and 10 years of experience, respectively) visually checked for segmentation or registration errors by overlaying each subject's nativespace-transformed ROI masks onto their T1-weighted images and modified the errors.

For radiomics analysis, all images were resampled to 1-mm isovoxels across all patients. A total of 107 radiomics features (including shape; first-order features; and second-order features consisting of gray level co-occurrence matrix, gray level run-length matrix, gray-level size zone matrix, gray level dependence matrix, and neighboring gray tone difference matrix (Supplementary Table 1)), were extracted from each hippocampus ROI. A total of 214 (107 features $\times$ two ROIs [right and left hippocampi]) radiomics features were obtained. The feature extraction was performed using an open-source Python-based package (PyRadiomics, version 2.0). (20)

\section{Statistical analysis}

For analysis of baseline characteristics and neuropsychological test scores, either Student's t-test or Mann Whitney's U test was used for continuous variables according to normality. Chi-square test was performed for categorical variables. All statistical analyses were performed using the statistical software R (version 3.6.0; R Foundation for Statistical Computing). Statistical significance was set at $p<0.05$.

\section{Radiomics feature selection and machine learning models with performance evaluation}

After normalization of all imaging features by z-score normalization, the least absolute shrinkage and selection operator (LASSO) with 10-fold cross validation was applied for feature selection. (21) LASSO is designed to avoid overfitting and is known to be suitable for analyzing high-dimensional datasets such as radiomics features. To evaluate whether radiomics improves prediction over models, three types of models were trained as follows: 1) a model based on radiomics features; 2) a clinical model based on demographics (age, sex, and education), APOE $\varepsilon 4$ status, and NP test results (MMSE, ADAS-cog, LM I, and LM II); and 3) a combined model based on radiomics features and clinical features. For 
classification, we applied the random forest (RF) algorithm. Hyperparameters were optimized by random search. In addition, to overcome data imbalance, each machine learning model was trained as follows: 1) without subsampling, 2) with synthetic minority over-sampling technique (SMOTE), and 3) with random over-sampling examples (ROSE). $(22,23)$ Thus, a total of 9 combinations of RF-based prediction models with different subsampling methods were trained and validated. The performance was evaluated in the training set with 10-fold cross-validation and validated in the test set. The area under the curve (AUC), accuracy, sensitivity, and specificity of each model were obtained. (24) The machine learning algorithms were trained and validated using Python 3 with Scikit-Learn library v0.21.2. The overall process is shown at Fig. 2.

\section{Results}

\section{Patient characteristics}

The baseline characteristics and NP test results of the $407 \mathrm{MCl}$ patients in the training and test sets are summarized in Table 1. In both the training and test sets, the CSF $A \beta_{42}+$ group was significantly older ( $p$ $=0.001$ and $p=0.003$ in the training and test set, respectively), had higher prevalence of APOE e4 carriers $(p<0.001$ and $p<0.001$ in the training and test set, respectively), showed higher scores in ADAS-cog $(p<$ 0.001 and $p=0.014$ in the training and test set, respectively) and lower LM I total $(p<0.001$ and $p=0.008$ in the training and test set, respectively) and LM II total scores $(p<0.001$ and $p=0.014$ in the training and test set, respectively) compared to the CSF $A \beta_{42}$ - group. 
Table 1

Clinical characteristics in the training and test sets.

\begin{tabular}{|c|c|c|c|c|c|c|c|}
\hline & \multicolumn{3}{|c|}{ Training set $(n=324)$} & \multicolumn{3}{|c|}{ Test set $(n=83)$} & \multirow{2}{*}{$\begin{array}{l}\text { p- } \\
\text { value } \\
+\end{array}$} \\
\hline & $\begin{array}{l}\operatorname{CSFA} \beta_{42}- \\
(n=123)\end{array}$ & $\begin{array}{l}\text { CSF A } \beta_{42}+ \\
(n=201)\end{array}$ & $\begin{array}{l}\text { p- } \\
\text { value* }\end{array}$ & $\begin{array}{l}\text { CSF A } \beta_{42}- \\
(n=31)\end{array}$ & 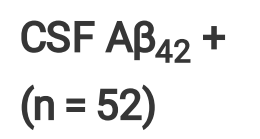 & $\begin{array}{l}\text { p- } \\
\text { value* }\end{array}$ & \\
\hline $\begin{array}{l}\text { Age } \\
\text { (mean } \pm \\
\text { SD) }\end{array}$ & $69.8 \pm 7.9$ & $72.5 \pm 7.0$ & 0.001 & $68.5 \pm 5.6$ & $72.4 \pm 7.3$ & 0.003 & 0.578 \\
\hline $\begin{array}{l}\text { Female } \\
(\%)\end{array}$ & $68(55.3)$ & $81(40.3)$ & 0.009 & $13(41.9)$ & $26(50.0)$ & 0.476 & 0.870 \\
\hline $\begin{array}{l}\text { Education } \\
\text { (years) }\end{array}$ & $16.0 \pm 2.6$ & $16.2 \pm 2.7$ & 0.319 & $16.5 \pm 2.4$ & $16.2 \pm 2.7$ & 0.668 & 0.667 \\
\hline $\begin{array}{l}\text { APOE } \varepsilon 4 \\
\text { carrier }\end{array}$ & $30(24.4)$ & $121(60.2)$ & $\dot{0} 001$ & 7 (22.6) & 36 (69.2) & $\dot{<} 0.001$ & 0.397 \\
\hline MMSE & $28.5 \pm 1.5$ & $27.7 \pm 1.9$ & $<.001$ & $28.8 \pm 1.2$ & $28.3 \pm 1.6$ & 0.182 & 0.052 \\
\hline ADAS-cog & $7.7 \pm 3.8$ & $10.4 \pm 4.7$ & $<.001$ & $7.1 \pm 3.6$ & $9.5 \pm 4.4$ & 0.014 & 0.178 \\
\hline $\begin{array}{l}\text { LM I total } \\
\text { score }\end{array}$ & $10.2 \pm 3.3$ & $8.8 \pm 3.5$ & $\dot{0} 001$ & $11.1 \pm 2.9$ & $9.3 \pm 2.9$ & 0.008 & 0.178 \\
\hline $\begin{array}{l}\text { LM II total } \\
\text { score }\end{array}$ & $7.9 \pm 2.7$ & $6.4 \pm 3.4$ & $<.001$ & $8.6 \pm 2.6$ & $6.7 \pm 3.2$ & 0.014 & 0.393 \\
\hline $\begin{array}{l}\text { LM II cue } \\
\text { score }\end{array}$ & $0.0 \pm 0.2$ & $0.2 \pm 0.4$ & 0.001 & $0.1 \pm 0.3$ & $0.2 \pm 0.4$ & 0.179 & 0.244 \\
\hline \multicolumn{8}{|c|}{ Data are presented as number of patients $(\%)$ or mean \pm SD. } \\
\hline \multicolumn{8}{|c|}{$\begin{array}{l}\text { * } p \text {-values were calculated using Student's t-test for continuous variables and chi-square test for } \\
\text { categorical variables, to compare subject characteristics between the CSF A } \beta_{42}-\text { and CSF } A \beta_{42}{ }^{+} \\
\text {groups in the training and test sets, respectively. }\end{array}$} \\
\hline \multicolumn{8}{|c|}{$\begin{array}{l}\text { † p-values were calculated using Student's t-test for continuous variables and chi-square test for } \\
\text { categorical variables, to compare subject characteristics between the training and test set. }\end{array}$} \\
\hline \multicolumn{8}{|c|}{$\begin{array}{l}\mathrm{A} \beta=\text { amyloid } \beta, A D A S=\text { Alzheimer's Disease Assessment Scale, } \mathrm{APOE}=\text { apolipoprotein } \mathrm{E}, \mathrm{CSF}= \\
\text { cerebrospinal fluid, } \mathrm{LM}=\text { logical memory, } \mathrm{MMSE}=\text { Mini-Mental State Exam }\end{array}$} \\
\hline
\end{tabular}

There were no differences in the clinical characteristics and NP test results between the training and test sets.

\section{Radiomics features and classification performance}


In the radiomics model, 33 radiomics features ( 17 from the right and 16 from the left hippocampus) were selected to predict the CSF A $\beta$ status in the training set (Supplementary Table 2). The selected features consisted of 7 shape features, 4 first-order features, and 22 second-order features (e.g., gray level runlength matrix, gray-level size zone matrix, gray level dependence matrix, and neighboring gray tone difference matrix). The AUCs ranged from 0.594 to 0.718 in the training set. The best performing radiomics model in the test set was RF with ROSE, with an AUC, accuracy, sensitivity, and specificity of 0.674 (95\% confidence interval [Cl]: $0.557-0.790), 65.1 \%, 34.2 \%$, and $91.1 \%$, respectively.

In the clinical model, patient sex, age, education (years), ADAS-cog, LM I total score, and APOE $\varepsilon 4$ status were included to predict the CSF A $\beta$ status in the training set. The AUCs ranged from 0.723 to 0.769 in the training set. The best performing radiomics model in the test set was RF with ROSE, with an AUC, accuracy, sensitivity, and specificity of 0.758 (95\% Cl: $0.656-0.861), 71.1 \%, 78.9 \%$, and $64.4 \%$, respectively.

In the combined model of radiomics and clinical features, 32 out of 33 radiomics features from the radiomics model were retained, and 5 out of 6 clinical features from the clinical model were retained (Supplementary Table 3). The features that dropped out from the LASSO procedure in the combined model compared to the radiomics and clinical models were 1 first-order feature (minimum) and patient sex, respectively. The AUCs ranged from 0.732 to 0.804 in the training set. The best performing radiomics model in the test set was RF with SMOTE, with an AUC, accuracy, sensitivity, and specificity of 0.823 (95\% Cl: $0.734-0.912), 77.1 \%, 63.2 \%$, and $88.9 \%$, respectively. The combined model showed higher performance than either radiomics (AUC 0.674) or clinical model (AUC 0.758) in the test set. The diagnostic performance of the test set of the three models is summarized in Table 2. 
Table 2

Diagnostic performance of each machine learning model in the test set for prediction of CSF A $\beta$ positivity.

\begin{tabular}{|c|c|c|c|c|}
\hline & AUC (95\% Cl) & Accuracy (\%) & Sensitivity (\%) & Specificity (\%) \\
\hline \multicolumn{5}{|l|}{ Model 1 (radiomics) } \\
\hline RF without subsampling & $0.633(0.512-0.754)$ & 61.4 & 36.8 & 82.2 \\
\hline RF with SMOTE & $0.656(0.537-0.775)$ & 61.4 & 36.8 & 82.2 \\
\hline RF with ROSE & $0.674(0.557-0.790)$ & 65.1 & 34.2 & 91.1 \\
\hline \multicolumn{5}{|l|}{ Model 2 (clinical) } \\
\hline RF without subsampling & $0.777(0.678-0.877)$ & 66.3 & 65.8 & 66.7 \\
\hline RF with SMOTE & $0.747(0.641-0.853)$ & 60.2 & 36.8 & 80.0 \\
\hline RF with ROSE & $0.758(0.656-0.861)$ & 71.1 & 78.9 & 64.4 \\
\hline \multicolumn{5}{|c|}{ Model 3 (radiomics + clinical) } \\
\hline RF without subsampling & $0.815(0.726-0.904)$ & 65.1 & 68.4 & 62.2 \\
\hline RF with SMOTE & $0.823(0.734-0.912)$ & 77.1 & 63.2 & 88.9 \\
\hline RF with ROSE & $0.814(0.722-0.906)$ & 72.3 & 73.7 & 71.1 \\
\hline
\end{tabular}

\section{Discussion}

In this study, we developed and validated a prediction model based on a combination of clinical and radiomics features that could predict $A \beta$ positivity based on CSF analysis at single subject level. The combined model involving both clinical and radiomic features showed the best performance (AUC: 0.823), followed by clinical model (AUC: 0.758), and the radiomics model (AUC: 0.674 ) in the test set, showing the utility and robustness of the combined model. These results indicate the independent contribution of radiomics and clinical features in identifying $\mathrm{MCl}$ with CSF $\mathrm{A} \beta$ pathology and the added value of the radiomics beyond the effects of clinical features.

Accumulation of $A \beta$ pathology is one of the hallmark pathologic characteristics of the AD continuum and precedes decades before the onset of cognitive symptoms. (6) Recently, many amyloid-modifying therapy trials in $A D$ subjects failed to show its effectiveness, (25-27) and one of the presumed reason for failure is the enrollment of subjects with clinical heterogeneity who did not have increased cerebral $A \beta$ plaques and were unlikely to have had AD pathology. (28) Therefore, the identification of $A \beta$ biomarkers via CSF $A \beta$ or PET is important to diagnose the AD continuum in both research and clinical settings. However, 
these biomarkers are not routinely acquired in clinics, owing to limited resource, high costs, and the need for invasive procedures. Therefore, practical methods to determine candidates for the amyloid biomarker test with commonly available clinical and MRI data may be helpful.

Compared to $\mathrm{MCl}$ subjects without amyloid pathology, those with amyloid pathology have significantly lower volumes in various brain regions including the hippocampus. (29-32) Previous studies have already attempted to predict the amyloid pathology using these MRI features in $\mathrm{MCl}$ patients. They mainly used volume features of the hippocampus and other $A D$ vulnerable structures, $(29,31,33)$ offering a fair degree of diagnostic performance. Predictive models that combined both MRI and clinical features showed good performance in identifying amyloid pathology of $\mathrm{MCl}$ patients. $(29-31,33)$ Consistent findings were found in the current study. However, the most previous studies were performed without proper validation in a test set, which may have led to overfitted results, especially in highdimensional datasets with machine learning studies. (34) A recent study applying data-driven algorithm with clinical features with validation showed an AUC of 0.71 at the test set, (35) showing only fair performance, unlike that in previous studies. This gives another line of evidence of potential overfitted results of the previous studies. Meanwhile, our model that integrated radiomics and clinical features showed good performance not only in the training set, but also in the test set. The robust predictive capacity of our combined models shows that it can help triage the subjects for more invasive and costly $A \beta$ testing.

Although previous radiomics studies in the neuroradiology field have mostly been focused on neurooncology, (36-40) there have also been several recent studies using radiomics analysis on T1-weighted images in AD. These studies have shown promising results not only in the diagnosis of AD but also in the prediction of disease progression. (41-44) Radiomics features may be prone to biological validation for their correlation with disease pathology. (45) This observation is based on the hypothesis that radiomics features, especially second-order features, capture the spatial variation in signal intensity that may reflect the deposition of $A \beta$ plaques. Further, it may extract different biological information from volume, $(43,46)$ which is the traditional imaging biomarker of $A D$.

Notably, nearly all the radiomics features were retained in the combined model after the LASSO procedure in our study. This suggests that most radiomics features harbor information independent from the clinical features, which may provide added value in predicting the CSF A $\beta$ status. However, the prediction of CSF $A \beta$ status by radiomics features alone was not optimal, confirming the importance of clinical features. Nonetheless, our results indicate that the added value of radiomics features over clinical features is a robust method.

Our study has several limitations. First, we only included the radiomics features of the hippocampus, as previous studies showed good performance using the hippocampus mask for the classification and prediction of AD. $(40,42,47,48)$ However, volume changes not only occur in the hippocampus, but also in other AD signature regions such as the entorhinal cortex and precuneus. (6) Thus, the radiomics prediction model could be improved by adding radiomic information of other anatomical structures. 
Further, whole brain investigation should be performed in future studies. Second, CSF A $\beta$ status was used as the gold standard for $A \beta$ positivity rather than PET imaging. It could be argued that the performance of the prediction model could be sensitive to the selection of the gold-standard method. However, the agreement between CSF and PET determinations of $A \beta$ positivity is very high, particularly in the intermediate ranges where thresholds for positivity typically lie. $(49,50)$

\section{Conclusions}

An MRI radiomics-based model can help predict CSF A $\beta_{42}$ status in $\mathrm{MCl}$ patients and can potentially triage the patients for the invasive and costly $A \beta$ test.

\section{Abbreviations}

$A \beta=$ amyloid $\beta, A D=$ Alzheimer's disease, $A D A S=$ Alzheimer's Disease Assessment Scale, $A D N I=$ Alzheimer's Disease Neuroimaging Initiative, $\mathrm{APOE}=$ apolipoprotein $\mathrm{E}, \mathrm{AUC}=$ area under the curve, $\mathrm{Cl}=$ confidence interval, CSF = cerebrospinal fluid, LASSO = least absolute shrinkage and selection operator, $\mathrm{LM}=$ Logical Memory, $\mathrm{MCl}=$ mild cognitive impairment, $\mathrm{MMSE}=$ Mini-Mental State Exam, MRI = magnetic resonance imaging, $\mathrm{PET}=$ positron emission tomography, $\mathrm{RF}=$ random forest, $\mathrm{ROSE}=$ random over-sampling example, SMOTE = synthetic minority over-sampling technique

\section{Declarations}

\section{Ethics approval and consent to participate}

We obtained data from the Alzheimer's Disease Neuroimaging Initiative database (adni.loni.usc.edu). The Alzheimer's Disease Neuroimaging Initiative was approved by the institutional review board at each site, and all participants gave their written consent.

\section{Consent for publication}

The Alzheimer's Disease Neuroimaging Initiative was approved by the institutional review board at each site, and all participants gave their written consent.

\section{Availability of data and materials}

The dataset analyzed in the current study are available in the Alzheimer's Disease Neuroimaging Initiative database. 


\section{Competing interests}

The authors declare that they have no competing interests.

\section{Funding}

None.

\section{Authors' contributions}

YWP analyzed and interpreted the patient demographics and clinical data and drafted the manuscript. DC performed radiomics and machine learning analysis of the dataset. MP conceptualized and designed the work and revised the manuscript. SJA acquired the dataset. SSA designed the radiomics framework. SHS revised the manuscript. SKL conceptualized the work. All authors read and approved the final manuscript.

\section{Acknowledgements}

None.

\section{References}

1. Mitchell AJ, Shiri-Feshki M. Rate of progression of mild cognitive impairment to dementia-metaanalysis of 41 robust inception cohort studies. Acta Psychiatrica Scandinavica. 2009;119(4):252-65.

2. McGeer PL, McGeer EG. The amyloid cascade-inflammatory hypothesis of Alzheimer disease: implications for therapy. Acta neuropathologica. 2013;126(4):479-97.

3. Tosun D, Joshi S, Weiner MW, Initiative AsDN. Neuroimaging predictors of brain amyloidosis in mild cognitive impairment. Annals of neurology. 2013;74(2):188-98.

4. McKhann GM, Knopman DS, Chertkow H, Hyman BT, Jack Jr CR, Kawas CH, et al. The diagnosis of dementia due to Alzheimer's disease: recommendations from the National Institute on AgingAlzheimer's Association workgroups on diagnostic guidelines for Alzheimer's disease. Alzheimer's \& dementia. 2011;7(3):263-9.

5. Park M, Moon W-J. Structural MR imaging in the diagnosis of Alzheimer's disease and other neurodegenerative dementia: current imaging approach and future perspectives. Korean journal of radiology. 2016;17(6):827-45.

6. Frisoni GB, Fox NC, Jack CR, Scheltens P, Thompson PM. The clinical use of structural MRI in Alzheimer disease. Nature Reviews Neurology. 2010;6(2):67-77. 
7. Gillies RJ, Kinahan PE, Hricak H. Radiomics: images are more than pictures, they are data. Radiology. 2015;278(2):563-77.

8. Folstein MF, Folstein SE, McHugh PR. "Mini-mental state": a practical method for grading the cognitive state of patients for the clinician. Journal of psychiatric research. 1975;12(3):189-98.

9. Mirra SS, Heyman A, McKeel D, Sumi S, Crain BJ, Brownlee L, et al. The Consortium to Establish a Registry for Alzheimer's Disease (CERAD): Part II. Standardization of the neuropathologic assessment of Alzheimer's disease. Neurology. 1991;41(4):479-.

10. Mohs RC. Administration and scoring manual for the Alzheimer's Disease Assessment Scale, 1994 revised edition. New York: The Mount Sinai School of Medicine. 1994.

11. Shaw LM, Vanderstichele H, Knapik-Czajka M, Figurski M, Coart E, Blennow K, et al. Qualification of the analytical and clinical performance of CSF biomarker analyses in ADNI. Acta neuropathologica. 2011;121(5):597-609.

12. Shaw LM, Vanderstichele H, Knapik-Czajka M, Clark CM, Aisen PS, Petersen RC, et al. Cerebrospinal fluid biomarker signature in Alzheimer's disease neuroimaging initiative subjects. Annals of neurology. 2009;65(4):403-13.

13. Jack Jr CR, Bernstein MA, Fox NC, Thompson P, Alexander G, Harvey D, et al. The Alzheimer's disease neuroimaging initiative (ADNI): MRI methods. Journal of Magnetic Resonance Imaging: An Official Journal of the International Society for Magnetic Resonance in Medicine. 2008;27(4):685-91.

14. Manjón JV, Coupé P. volBrain: an online MRI brain volumetry system. Frontiers in neuroinformatics. 2016;10:30.

15. Næss-Schmidt E, Tietze A, Blicher JU, Petersen M, Mikkelsen IK, Coupé P, et al. Automatic thalamus and hippocampus segmentation from MP2RAGE: comparison of publicly available methods and implications for DTI quantification. International journal of computer assisted radiology and surgery. 2016;11(11):1979-91.

16. Romero JE, Coupé P, Manjón JV. HIPS: A new hippocampus subfield segmentation method. Neurolmage. 2017;163:286-95.

17. Avants BB, Tustison N, Song G. Advanced normalization tools (ANTS). Insight j. 2009;2(365):1-35.

18. Nyúl LG, Udupa JK. On standardizing the MR image intensity scale. Magnetic Resonance in Medicine: An Official Journal of the International Society for Magnetic Resonance in Medicine. 1999;42(6):1072-81.

19. Coupé P, Manjón JV, Chamberland M, Descoteaux M, Hiba B. Collaborative patch-based superresolution for diffusion-weighted images. Neurolmage. 2013;83:245-61.

20. van Griethuysen JJ, Fedorov A, Parmar C, Hosny A, Aucoin N, Narayan V, et al. Computational radiomics system to decode the radiographic phenotype. Cancer research. 2017;77(21):e104-e7.

21. Tibshirani R. Regression shrinkage and selection via the lasso. Journal of the Royal Statistical Society Series B (Methodological). 1996:267-88. 
22. Chawla NV, Bowyer KW, Hall LO, Kegelmeyer WP. SMOTE: synthetic minority over-sampling technique. Journal of artificial intelligence research. 2002;16:321-57.

23. Provost $F$, editor Machine learning from imbalanced data sets 101. Proceedings of the AAAl'2000 workshop on imbalanced data sets; 2000.

24. Saito T, Rehmsmeier M. The precision-recall plot is more informative than the ROC plot when evaluating binary classifiers on imbalanced datasets. PloS one. 2015;10(3):e0118432.

25. Salloway S, Sperling R, Fox NC, Blennow K, Klunk W, Raskind M, et al. Two phase 3 trials of bapineuzumab in mild-to-moderate Alzheimer's disease. New England Journal of Medicine. 2014;370(4):322-33.

26. Doody RS, Thomas RG, Farlow M, Iwatsubo T, Vellas B, Joffe S, et al. Phase 3 trials of solanezumab for mild-to-moderate Alzheimer's disease. New England Journal of Medicine. 2014;370(4):311-21.

27. Coric V, Salloway S, van Dyck CH, Dubois B, Andreasen N, Brody M, et al. Targeting prodromal Alzheimer disease with avagacestat: a randomized clinical trial. JAMA neurology. 2015;72(11):132433.

28. Brashear HR. Comment: Age effects on clinical trial results in Alzheimer dementia. Neurology. 2015;84(11):1126-.

29. Ten Kate M, Redolfi A, Peira E, Bos I, Vos SJ, Vandenberghe R, et al. MRI predictors of amyloid pathology: results from the EMIF-AD Multimodal Biomarker Discovery study. Alzheimer's research \& therapy. 2018;10(1):100.

30. Apostolova L, Hwang K, Kohannim O, Jack C, Shaw L, Trojanowski J, et al. Predicting brain amyloidosis in $\mathrm{MCl}$ using clinical, cognitive, imaging and peripheral blood protein measures. Alzheimer's \& Dementia: The Journal of the Alzheimer's Association. 2012;8(4):S747-S8.

31. Apostolova LG, Hwang KS, Avila D, Elashoff D, Kohannim O, Teng E, et al. Brain amyloidosis ascertainment from cognitive, imaging, and peripheral blood protein measures. Neurology. 2015;84(7):729-37.

32. Huijbers W, Mormino EC, Schultz AP, Wigman S, Ward AM, Larvie M, et al. Amyloid- $\beta$ deposition in mild cognitive impairment is associated with increased hippocampal activity, atrophy and clinical progression. Brain. 2015;138(4):1023-35.

33. Ezzati A, Harvey DJ, Habeck C, Golzar A, Qureshi IA, Zammit AR, et al. Predicting Amyloid- $\beta$ Levels in Amnestic Mild Cognitive Impairment Using Machine Learning Techniques. Journal of Alzheimer's Disease. 2019(Preprint):1-9.

34. Dietterich T. Overfitting and undercomputing in machine learning. ACM computing surveys (CSUR). 1995;27(3):326-7.

35. Maserejian N, Bian S, Wang W, Jaeger J, Syrjanen JA, Aakre J, et al. Practical algorithms for amyloid $\beta$ probability in subjective or mild cognitive impairment. Alzheimer's \& Dementia: Diagnosis, Assessment \& Disease Monitoring. 2019;11:180.

36. Park YW, Choi YS, Ahn SS, Chang JH, Kim SH, Lee S-K. Radiomics MRI phenotyping with machine learning to predict the grade of lower-grade gliomas: a study focused on nonenhancing tumors. 
Korean journal of radiology. 2019;20(9):1381-9.

37. Park YW, Han K, Ahn SS, Choi Y, Chang J, Kim S, et al. Whole-tumor histogram and texture analyses of DTI for evaluation of IDH1-mutation and 1p/19q-codeletion status in World Health Organization grade II gliomas. American Journal of Neuroradiology. 2018;39(4):693-8.

38. Park YW, Oh J, You SC, Han K, Ahn SS, Choi YS, et al. Radiomics and machine learning may accurately predict the grade and histological subtype in meningiomas using conventional and diffusion tensor imaging. European radiology. 2019;29(8):4068-76.

39. Park CJ, Choi YS, Park YW, Ahn SS, Kang S-G, Chang J-H, et al. Diffusion tensor imaging radiomics in lower-grade glioma: improving subtyping of isocitrate dehydrogenase mutation status. Neuroradiology. 2019:1-8.

40. Kickingereder P, Burth S, Wick A, Götz M, Eidel O, Schlemmer H-P, et al. Radiomic profiling of glioblastoma: identifying an imaging predictor of patient survival with improved performance over established clinical and radiologic risk models. Radiology. 2016;280(3):880-9.

41. Feng F, Wang P, Zhao K, Zhou B, Yao H, Meng Q, et al. Radiomic features of hippocampal subregions in Alzheimer's disease and amnestic mild cognitive impairment. Frontiers in aging neuroscience. 2018;10.

42. Chaddad A, Desrosiers C, Niazi T. Deep radiomic analysis of MRI related to Alzheimer's disease. IEEE Access. 2018;6:58213-21.

43. Sørensen L, Igel C, Liv Hansen N, Osler M, Lauritzen M, Rostrup E, et al. Early detection of Alzheimer's disease using MRI hippocampal texture. Human brain mapping. 2016;37(3):1148-61.

44. Feng F, Wang $P$, Zhao K, Zhou B, Yao H, Meng Q, et al. Radiomic features of hippocampal subregions in Alzheimer's disease and amnestic mild cognitive impairment. Frontiers in aging neuroscience. 2018;10:290.

45. Park JE, Kim HS, Kim D, Park SY, Kim JY, Cho SJ, et al. A systematic review reporting quality of radiomics research in neuro-oncology: toward clinical utility and quality improvement using highdimensional imaging features. BMC cancer. 2020;20(1):1-11.

46. Cai J-H, He Y, Zhong X-L, Lei H, Wang F, Luo G-H, et al. Magnetic Resonance Texture Analysis in Alzheimer's disease. Academic Radiology. 2020.

47. Luk CC, Ishaque A, Khan M, Ta D, Chenji S, Yang Y-H, et al. Alzheimer's disease: 3-Dimensional MRI texture for prediction of conversion from mild cognitive impairment. Alzheimer's \& Dementia: Diagnosis, Assessment \& Disease Monitoring. 2018;10:755-63.

48. Martínez-Torteya A, Rodríguez-Rojas J, Celaya-Padilla JM, Galván-Tejada JI, Treviño V, Tamez-Peña $J G$, editors. MRI signal and texture features for the prediction of $\mathrm{MCl}$ to Alzheimer's disease progression. Medical Imaging 2014: Computer-Aided Diagnosis; 2014: International Society for Optics and Photonics.

49. Leuzy A, Chiotis K, Hasselbalch SG, Rinne JO, de Mendonça A, Otto M, et al. Pittsburgh compound B imaging and cerebrospinal fluid amyloid- $\beta$ in a multicentre European memory clinic study. Brain. 2016;139(9):2540-53. 
50. Leuzy A, Carter SF, Chiotis K, Almkvist O, Wall A, Nordberg A. Concordance and diagnostic accuracy of [11C] PIB PET and cerebrospinal fluid biomarkers in a sample of patients with mild cognitive impairment and Alzheimer's disease. Journal of Alzheimer's Disease. 2015;45(4):1077-88.

\section{Figures}

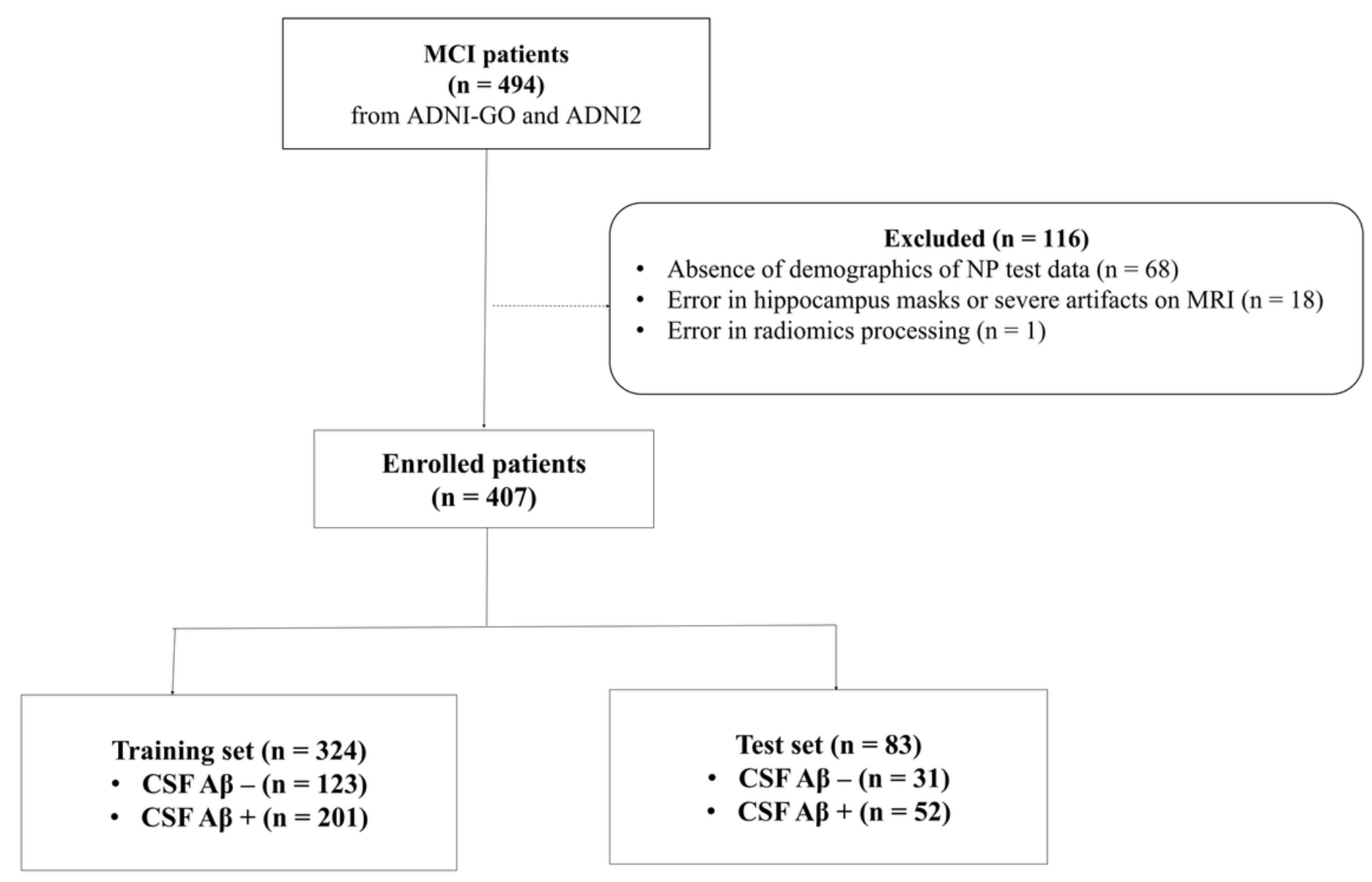

Figure 1

Patient inclusion flowchart. 


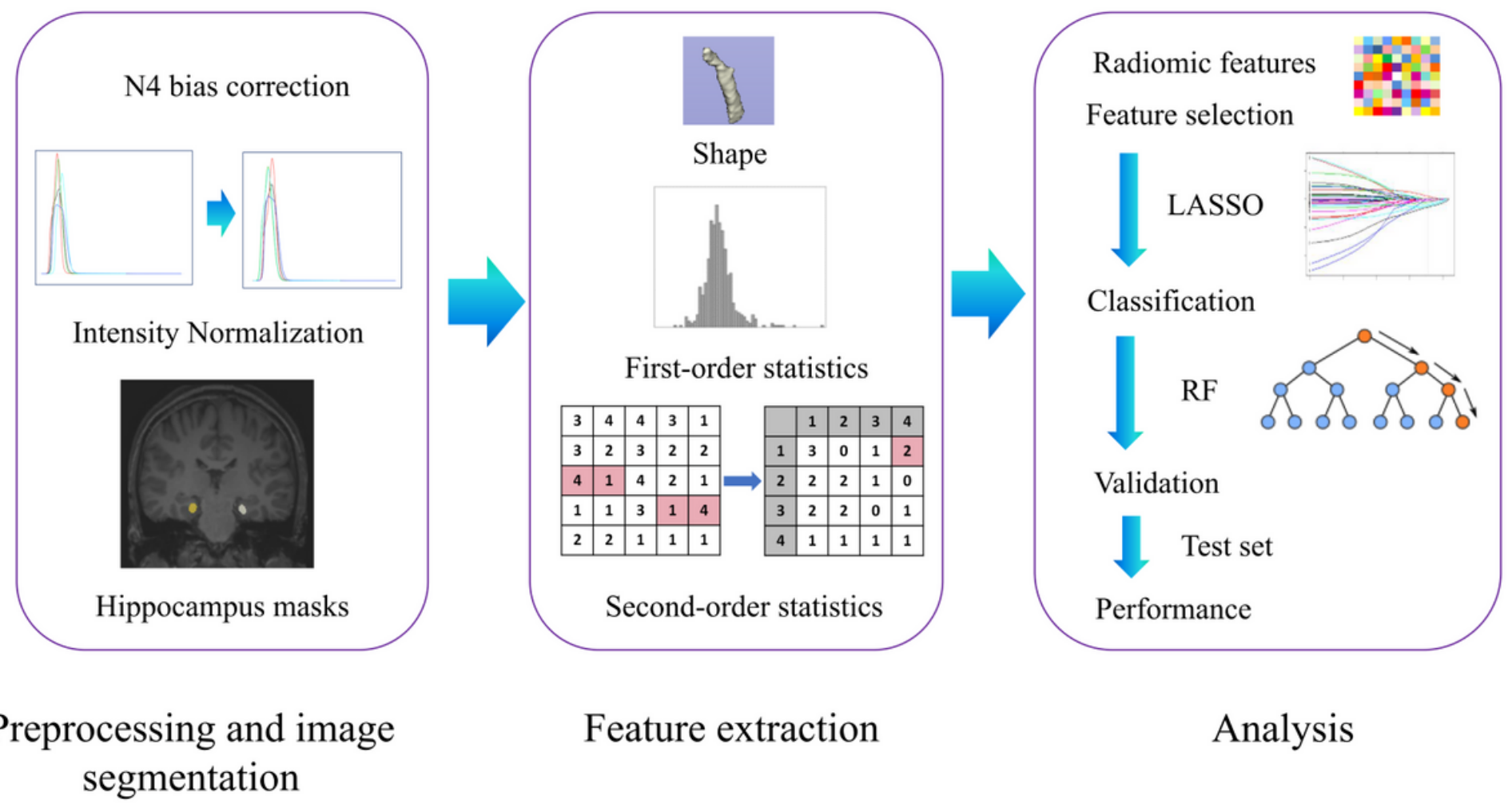

Figure 2

Workflow of image processing, radiomics feature extraction, and machine learning. LASSO = least absolute shrinkage and selection operator, $\mathrm{RF}=$ random forest

\section{Supplementary Files}

This is a list of supplementary files associated with this preprint. Click to download.

- 20200224ARTSupplementaryMaterial.docx 